\title{
EMC ASPECTS OF TURBULENCE HEATING OBSERVER (THOR) SPACECRAFT
}

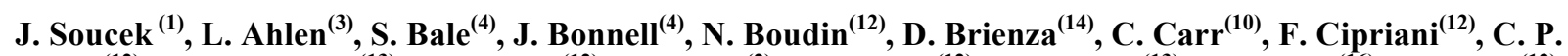
Escoubet $^{(12)}$, A. Fazakerley ${ }^{(13)}$, M. Gehler ${ }^{(12)}$, V. Génot ${ }^{(2)}$, A. Hilgers ${ }^{(12)}$, B. Hanock ${ }^{(13)}$, G. Jannet ${ }^{(16)}$, A. Junge ${ }^{(12)}$, Y. Khotyaintsev $^{(3)}$, J. De Keyser ${ }^{(17)}$, H. Kucharek ${ }^{(15)}$, R. Lan ${ }^{(1)}$, B. Lavraud ${ }^{(2)}$, F. Leblanc ${ }^{(7)}$, W. Magnes ${ }^{(11)}$, M.

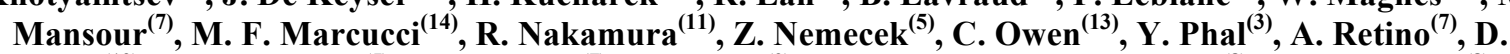
Rodgers $^{(12)}$, J. Safrankova ${ }^{(5)}$, F. Sahraoui ${ }^{(7)}$, R. Vainio ${ }^{(9)}$, R. Wimmer-Schweingruber ${ }^{(8)}$, J. Steinhagen ${ }^{(8)}$, A. Vaivads $^{(3)}$, A. Wielders ${ }^{(12)}$, A. Zaslavsky ${ }^{(6)}$

(1) Institute of Atmospheric Physics, Czech Academy of Science, Prague, Czech Republic, Email: soucek@ufa.cas.cz, (2) IRAP, Toulouse, France (3) Swedish Institute of Space Physics, Uppsala, Sweden, (4) Space Science Laboratory, UCB, USA (5) Charles University, Prague, Czech Republic, (6) LESIA, Paris Observatory, Meudon, France (7) Laboratory of Plasma Physics, Paris, France (8) University of Kiel, Kiel, Germany (9) University of Turku, Turku, Finland, (10) Imperial College, London UK, (11) Space Research Institute, Austrian Academy of Sciences, Graz, Austria (12) ESA-ESTEC, Noordwijk, The Netherlands (13) Mullard Space Science Laboratory, UCL, Dorking, UK (14) IAPS, Rome, Italy (15) University of New Hampshire, USA (16) LPC2E, Orleans, France (17) BIRA-IASB, Brussels, Belgium

\section{ABSTRACT}

Turbulence Heating ObserveR (THOR) is a spacecraft mission dedicated to the study of plasma turbulence in near-Earth space. The mission is currently under study for implementation as a part of ESA Cosmic Vision program. THOR will involve a single spinning spacecraft equipped with state of the art instruments capable of sensitive measurements of electromagnetic fields and plasma particles. The sensitive electric and magnetic field measurements require that the spacecraftgenerated emissions are restricted and strictly controlled; therefore a comprehensive EMC program has been put in place already during the study phase. The THOR study team and a dedicated EMC working group are formulating the mission EMC requirements state of its EMC requirements.

\section{THOR mission}

Turbulence Heating ObserveR (THOR) is a candidate for an ESA Cosmic Vision medium class mission. It is one of the three candidates selected for a study for the M4 slot. If selected, it will be the first mission ever flown in space dedicated to plasma turbulence. It will explore the kinetic plasma processes that determine the basic plasma heating and particle energization processes, their efficiency for different plasma species and their relative importance in different turbulent regimes. THOR will provide closure of these fundamental astrophysical questions by making detailed in situ measurements of the closest available dilute and

\begin{tabular}{|l|l|l|l|l|}
\hline Instrument & Description & Measured quantity & Range & Max. cadence \\
\hline MAG & Fluxgate magnetometer & Magnetic field & DC-50 Hz & $128 \mathrm{sps}$ \\
\hline SCM & Search coil magnetometer & Magnetic field & $1 \mathrm{~Hz}-200 \mathrm{kHz}$ & $524 \mathrm{ksps}$ \\
\hline EFI & $2 \times$ double probes, $3 \times$ boom & Electric field & DC-200 kHz & $524 \mathrm{ksps}$ \\
\hline FAR & Faraday cup & Fast ion moments & & $32 \mathrm{sps}$ \\
\hline FWP & EM field and wave receiver & Fields and waves & & $524 \mathrm{ksps}$ \\
\hline TEA & Electron spectrometer & Electron distribution & $1 \mathrm{eV}-30 \mathrm{keV}$ & $5 \mathrm{~ms}$ \\
\hline IMS & Ion spectrometer with TOF & Ion distribution & $5 \mathrm{eV}-40 \mathrm{keV} / \mathrm{q}$ & $150 \mathrm{~ms}$ \\
\hline CSW & Electrostatic analyser & Cold solar wind ions & $0.2-20 \mathrm{keV} / \mathrm{q}$ & $150 \mathrm{~ms}$ \\
\hline EPE & Solid state detector & Energetic particles & $\begin{array}{l}\mathrm{e}-: 20 \mathrm{keV}-700 \mathrm{keV} \\
\mathrm{i}+: 20 \mathrm{keV}-8 \mathrm{MeV}\end{array}$ & $7.5 \mathrm{~s}$ \\
\hline PPU & & & & \\
\end{tabular}

Table 1: THOR payload summary

already in the earliest phase of the project to avoid later delays and cost increases related to EMC. This article introduces the THOR mission and reviews the current turbulent magnetized plasmas at unprecedented temporal and spatial resolution. THOR will focus on particular regions: solar wind, Earth's bow shock and 
interplanetary shocks, and compressed solar wind regions downstream of shocks - the magnetosheath.

The THOR spacecraft will carry state of the art instruments tailored to explore plasma energization in turbulence via simultaneous measurements of both the turbulent electromagnetic field fluctuations and the signatures of plasma heating and acceleration in the velocity distribution functions of electrons and ions. The payload will be composed of the 10 instruments listed in Table 1

In-situ plasma measurements generally benefit from spinning spacecraft platforms. THOR will be a 2-rpm spinning satellite with the spin axis pointed at the Sun. This configuration is optimal for electric field measurements by double sphere probes mounted on 50 meter long wire booms. The orbit of THOR will evolve over the course of its 3.5 year nominal mission, starting with an apogee of 15 Earth radii to study the bow shock and gradually increasing the apogee to over 50 Earth radii to observe the pristine solar wind. For details on THOR mission and payload see the THOR proposal which can be downloaded from http://thor.irfu.se.

To reliably observe the weak electromagnetic fields as required by the science objectives and to ensure that measurements of charged particles are not perturbed by spacecraft generated fields, a comprehensive EMC program must be a part of the mission development. An EMC working group has been set up already during the study phase and charged with the task of formulating THOR EMC requirements.

\section{AC ELECTRIC AND MAGNETIC FIELD REQUIREMENTS}

A significant aspect of the THOR EMC program are the requirements imposed on $\mathrm{AC}$ electric and magnetic field emissions from the spacecraft in the low frequency range (below $200 \mathrm{kHz}$ ). The natural phenomena targeted by THOR, such as plasma waves and electromagnetic field oscillations associated with turbulence are observed in this frequency range and some of the oscillations are extremely faint. Requirements are formulated in spectral domain, for broadband noise and narrowband emissions separately. While broadband emissions cannot be removed from scientific data efficiently and must thus be eliminated, stable narrowband spectral spikes can be removed from the data by post-processing and higher amplitudes are thus acceptable.

The red line in Figure 1 shows the limit imposed by THOR requirements on the spacecraft magnetic emissions. This curve follows the expected performance of THOR search coil magnetometer (green line) which improves on the heritage instrument STAFF from the
Cluster spacecraft [3] (magenta line). While the EMC requirements are stricter than in the case of Cluster at the location of the sensor, when the longer booms of THOR are taken into account and the magnetic field is re-scaled appropriately, the THOR EMC requirement is actually similar to that of Cluster. The black dashed line shows the THOR requirement level re-scaled to Cluster boom length for easy comparison with the analogous Cluster requirement (blue) and performance obtained in space (magenta). This assumes boom length of 6.3 meters for THOR.

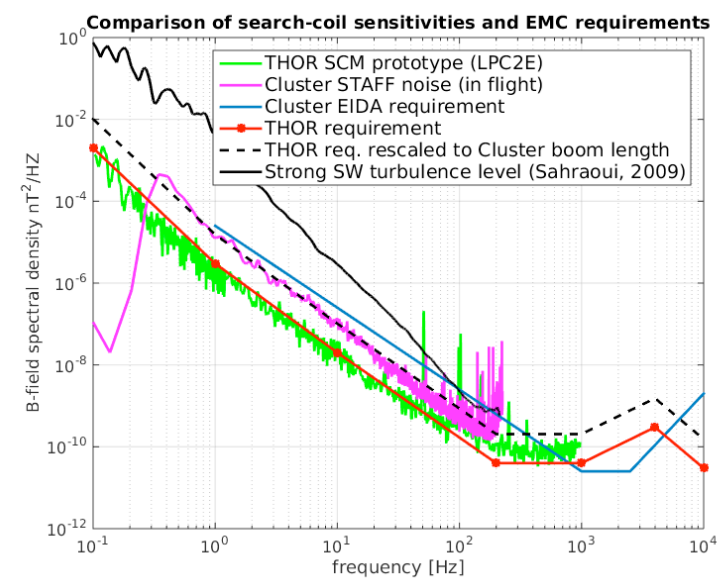

Figure 1 Comparison of THOR AC magnetic field requirement (red line) and performance of existing search coil magnetometers. Black line shows a typical turbulence level in the solar wind (from [2]).

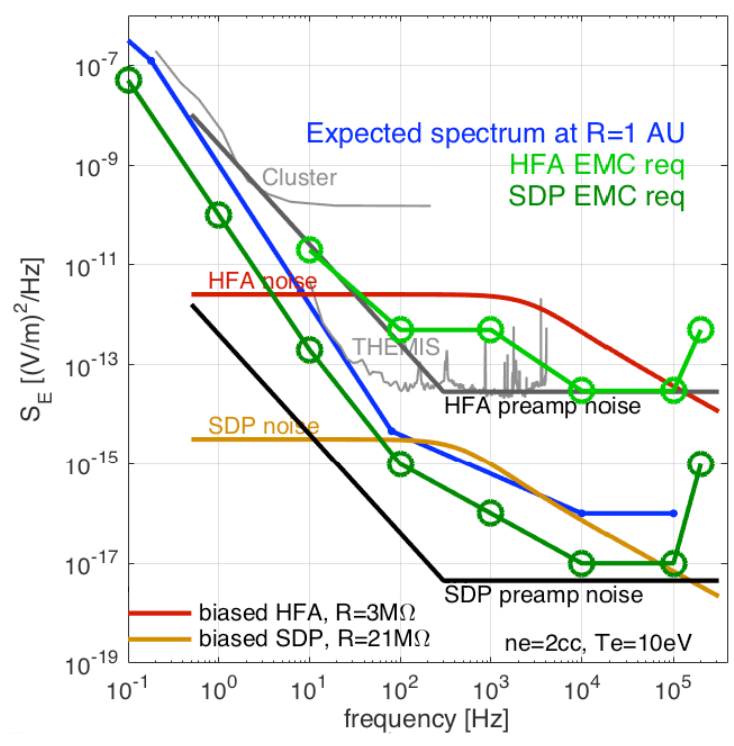

Figure 2 THOR AC electric field requirements compared with solar wind E-field levels expected for THOR and those measured by heritage instruments.

Figure 2 shows analogous requirements for spacecraft generated electric fields required for unperturbed measurements of electric fields associated with 
turbulence at lower frequencies and plasma thermal noise at higher frequencies. The limit is imposed on the radiated electric field as seen by THOR double probe antennas (SDP) in the spin plane and $2.5 \mathrm{~m}$ tip-to-tip triaxial dipole antennas (HFA) mounted on a boom. The black lines show the expected instrument noise for the double probe antennas (SDP) and dipole antennas (HFA). The red and orange lines show the expected observed noise floor in the solar wind, including shot noise and effect of the plasma environment. Green lines show the actual EMC requirements. Over-plotted in grey are also actual solar wind spectra from Cluster and THEMIS double probe experiments, showing performance limitations of the heritage instruments.

The curves in Figures 1 and 2 set the limit for broadband spacecraft emissions. Narrowband spectral spikes, such DC-DC converter interference, can be allowed to exceed this level by up to $30 \mathrm{~dB}$. However, it must be ensured that these spikes can be effectively removed from the data by digital post-processing. For this reason, the spikes exceeding the requirement must be sufficiently stable in frequency that they remain within the same bin of 2048 point discrete Fourier transform (DFT). The spikes also need to be limited to at most $2 \%$ of each frequency decade.

\section{FORMULATION AND VERIFICATION OF REQUIREMENTS}

THOR EMC requirements are often given in terms of spectral density of electric and magnetic field expressed either in $(\mathrm{V} / \mathrm{m})^{\wedge} 2 / \mathrm{Hz}$ or $\mathrm{nT}^{\wedge} 2 / \mathrm{Hz}$. However, all THOR instruments perform data acquisition in time domain and the spectral quantities are derived from the sampled time series via digital processing.

Verification of the requirements shall be performed by direct tests on an assembled spacecraft in an electromagnetically clean facility or numerical modelling. In particular for the AC magnetic requirements, very sensitive sensors, exceeding the performance of off the shelf instruments, might be needed to perform the measurement. In such a case, the easiest solution is to use the actual instrument, or its engineering model, mounted in its proper position on the spacecraft as a means of EMC verification. Alternatively, the fields can be measured closer to the spacecraft and the magnitude extrapolated to the sensor location; this will probably be the method of choice for AC electric field measurements. This verification by ground tests shall be complemented by modelling or estimation of induced currents and fields which cannot be reasonably simulated in ground tests.

It is important that during EMC tests the measurements and spectral calculations are performed in a manner consistent with data acquisition by THOR payload. For
AC electromagnetic measurements, this includes sampling of the measurement in time domain and calculation of averaged spectra using DFT. This is automatically satisfied if the verification is performed by the actual payload instrument, in other cases the tests have to be designed respecting the payload measurement techniques. A commented version of the EMC requirement will be provided to the industrial contractor, describing the procedure, bandwidth and averaging used in spectral calculation in order to achieve consistent results.

\section{OTHER EMC REQUIREMENTS}

Since THOR shall provide accurate low frequency magnetic field measurements in the solar wind, where the typical natural magnetic field is $5 \mathrm{nT}$, spacecraft DC magnetic field needs to be controlled and characterized on the ground. The magnetometers will be mounted on long booms, reducing the impact of spacecraft magnetic field, but the magnitude of the system magnetic field at the position of the MAG outboard sensor shall be less than $2 \mathrm{nT}$ (for comparison, an analogous requirement for Cluster spacecraft specified a limit of $0.25 \mathrm{nT}$ ) and limits on the slow variations of the field (on the time scales of hours to seconds) are imposed. A magnetic model of the spacecraft will need to be constructed in order to predict the magnetic field at MAG outboard sensor and magnetic budgets shall be allocated to spacecraft subsystems and payload units. Before launch, the requirements shall be verified by direct tests at a magnetically clean facility and modelling of induced fields.

The THOR payload includes several detectors mounted on spacecraft body which register charged plasma particles entering the apertures of the detectors. The instruments must be able to sample the particle energy distribution down to very low energies (a few electronvolts) and this measurement can be perturbed by deflection of low energy particles by electrostatic and magnetic field of the spacecraft. Limits are thus imposed on the magnetic field integrated along the line of sight of particle instruments to avoid this deflection.

Spacecraft charging due to photoelectron fluxes, and in particular differential surface charging, can impact not only observations of particle velocity distributions, but also electric field measurements. THOR will implement active spacecraft potential control that should keep the spacecraft potential within predefined limits $(<10 \mathrm{~V})$. This device will use the same principle as ASPOC instruments [4] previously flown on several spacecraft including Cluster, Double Star and MMS. These devices reduce the positive potential via emission of Indium ions. The requirements on spacecraft charging cannot be effectively verified on the ground and numerical simulations (e.g. using SPIS software) shall be used. 
Furthermore, it is required that the spacecraft surface is fully conductive and that plasma analyzers are shielded by grounded grids, keeping the voltage between any two points on the spacecraft surface below $1 \mathrm{~V}$.

\section{IMPACT ON THE SPACECRAFT DESIGN}

Non-conformance issues from past missions were identified and studied, and an overall grounding scheme along with instrument level grounding was outlined. We prefer to have distributed single point grounding for the spacecraft. The grounding scheme is such that there is no return current through the chassis. To minimize the common ground coupling between instruments, it is preferred to have the spacecraft structure to be highly conductive (carbon fibre to be avoided).

All electronics of electromagnetic field instruments will be integrated in a single shielded electronics box (The Fields and Waves Processor instrument - FWP) which shall minimize the interference from the spacecraft toward payload electronics. At the unit level for FWP, a backplane is introduced providing a low impedance ground for connecting the individual sub-systems. The backplane is then connected at multiple points to the chassis of the FWP box in order to have minimum grounding electrical length.

The THOR spacecraft shall provide a master clock signal to the payload and platform units. This master clock shall be used to synchronize switching DC-DC converters and also instrument sampling clocks via phase locked loops. Having the major sources of interference phase synchronized to the measurements shall greatly facilitate the removal of spurious emissions from scientific data.

\section{SUMMARY}

THOR EMC requirements are formulated based on heritage from previous ESA missions carrying plasma and electromagnetic field instruments. Cluster spacecraft are being used as a reference design with extremely good magnetic cleanliness. Experience from Solar Orbiter and JUICE spacecraft is also taken into account. While THOR EMC requirements are certainly challenging, it can be shown than in many aspects they are not stricter than those of previous missions.

The requirements are currently being formulated by THOR EMC working group and the details and levels presented in this article must be considered preliminary. The ultimate objective of this effort is to prepare complete EMC requirements for the industrial contractors building the spacecraft, including detailed guidelines how the requirements can be verified. If this activity is successful, THOR approach to EMC can demonstrate that with proper planning and management of this aspect of the mission, a clean spacecraft can be built at modest additional cost and without an impact on schedule.

\section{REFERENCES}

1. Escoubet, C. P., Russel, C. T., and Schmidt, R., (1997), The Cluster and Phoenix missions, Kluwer Academic Publishers, ISBN 0-7923-4411-1.

2. Sahraoui, F., Goldstein, M. L., Robert, P., and Khotyaintsev, Yu (2009), Physical Review Letters, 102,231102

3. Cornilleau-Wehrlin, N., Chaveau, P., Louis, S., et al. (1997), The Cluster Spatio-Temporal Analysis of Field Fluctuations (STAFF) experiment, Space Science Reviews 79: 107-136.

5. Riedler, W., K. Torkar, K., et al. (1997), Active Spacecraft Potential Control, Space Science Reviews 79: 271-302

5. Solar Orbiter Experiment Interface Document (EID-A). SOL-EST-RCD-00050, Issue 5, Rev 0 $16^{\text {th }}$ March 2015. 